\title{
Pure or mixed-type classical invasive lobular carcinoma might show different imaging features
}

\author{
Kadri Altundag ${ }^{1}$ (D)
}

Received: 12 August 2018 / Accepted: 17 August 2018 / Published online: 22 August 2018

(c) Springer Science+Business Media, LLC, part of Springer Nature 2018

Dear Editor,

I want to congratulate Yeap and their colleagues for their article [1] in which they compared the imaging features of pleomorphic (pILC) and classical (cILC) invasive lobular carcinoma. They reported that there were differences in the imaging features between pILC and cILC which reflect the more aggressive nature of pILC. However, the authors did not describe whether cILC is pure or mixed type. Because some cases with cILC also carry features of infiltrative ductal carcinoma (IDC), the definition of cases with cILC may be important whether they are pure ILC or mixed type [2]. Existence of IDC within cILC tumor might show different imaging features compared to pILC or pure pILC. This issue merits further investigation.

Kadri Altundag

altundag66@yahoo.com

1 MKA Breast Cancer Clinic, Tepe Prime, Cankaya, 06800 Ankara, Turkey
Funding The author has not received any grants.

\section{Compliance with ethical standards}

Conflict of interest I have no conflict of interest to declare.

Ethical approval This article does not contain any studies with human participants or animals performed by any of the authors.

\section{References}

1. Yeap PM, Evans A, Purdie CA, Jordan LB, Vinnicombe SJ (2018) A comparison of the imaging features of pleomorphic and classical invasive lobular carcinoma. Breast Cancer Res Treat. https:// doi.org/10.1007/s10549-018-4914-8

2. Bulut N, Dede DS, Sari E, Altundag K (2008) Pure or mixed-type invasive lobular carcinoma? Hum Pathol 39(7):1121 